\title{
THE
}

$1-1980$

\section{Temperature and Field Dependence of Autocorrelation Functions for the One-Dimensional Heisenberg Antiferromagnet}

Gerhard Müller

University of Rhode Island, gmuller@uri.edu

Hans Beck

Follow this and additional works at: https://digitalcommons.uri.edu/phys_facpubs

Terms of Use

All rights reserved under copyright.

\section{Citation/Publisher Attribution}

Müller, G., \& Beck, H. (1980). Temperature and field dependence of autocorrelation functions for the onedimensional Heisenberg antiferromagnet. J. Magnetism and Magn. Materials, 15-18(2), 689-690. doi: 10.1016/0304-8853(80)90722-2

Available at: http://www.sciencedirect.com/science/article/pii/0304885380907222

This Article is brought to you for free and open access by the Physics at DigitalCommons@URI. It has been accepted for inclusion in Physics Faculty Publications by an authorized administrator of DigitalCommons@URI. For more information, please contact digitalcommons-group@uri.edu. 


\title{
Temperature and Field Dependence of Autocorrelation Functions for the One-Dimensional Heisenberg Antiferromagnet
}

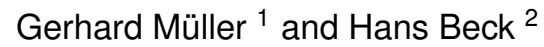 \\ ${ }^{1}$ Institut für Physik, Universität Basel, $\mathrm{CH}-4056$ Basel, Switzerland \\ 2 Institut de Physique de l'Université, $\mathrm{CH}-2000$ Neuchâtel, Switzerland
}

We present analytical and numerical results for the low frequency autocorrelation function of the I-d $s=\frac{1}{2}$ Heisenberg antiferromagnet at low temperature and various fields. Our results are in good agreement with NMR data.

The low temperature magnetic properties of the copper salts $\mathrm{CuSO}_{4} \cdot 5 \mathrm{H}_{2} \mathrm{O}, \mathrm{CuSeO}_{4} \cdot 5 \mathrm{H}_{2} \mathrm{O}$, $\mathrm{CuBeF}_{4} \cdot 5 \mathrm{H}_{2} \mathrm{O}$ can be described by treating the crystal as a system of loosely coupled $s=\frac{1}{2}$ antiferromagnetic chains [1] with Hamiltonian

$$
\mathcal{H}=\sum_{l=1}^{N} \mathbf{S}(l) \cdot \mathbf{S}(l+1)-h \sum_{l=1}^{N} S_{z}(l) .
$$

Recently [l] the dynamics of the $\mathrm{Cu}$ spins belonging to such chains has been investigated by NMR, performed on the protons of the $\mathrm{H}_{2} \mathrm{O}$-molecules. Basically the inverse "spin-lattice" relaxation time $T_{1}$ characterizing the influence of the $\mathrm{Cu}$ spins on the proton moments (due to dipolar interactions) is determined by the dynamical autocorrelation functions of the chain spins [1]:

$$
\phi_{\alpha \alpha}(\omega) \equiv \int d t e^{i \omega t}\left\langle S_{\alpha}(t) S_{\alpha}(0)\right\rangle, \quad 1 / T_{1}=A_{z} \phi_{z z}\left(\omega_{\mathrm{N}}\right)+A_{x} \phi_{x x}\left(\omega_{\mathrm{N}}\right) .
$$

$A_{x}$ and $A_{z}$ depend on geometry. The nuclear Larmor frequency $\omega_{\mathrm{N}}$ is small compared to the exchange constant (our unit of energy) and will therefore be replaced by zero.

In order to calculate $T_{1}^{-1}$ we need the functions $\phi_{z z}(0)$ and $\phi_{x x}(0)$ for the Hamiltonian (1) at various temperatures and fields. $\phi_{z z}(0)$ was calculated [2] assuming the low-temperature dynamics to be governed by a single branch of non-interacting, sharp spin-waves. This assumption leads directly to a divergence of $\phi_{z z}(0)$ for $T=0$. On the other hand, experiments on $s=\frac{1}{2}$ systems $[1,2]$ point to a finite limit of $\phi_{z z}(0)$. Recently the field dependence of $T_{1}$ for various $T$ has been measured [3] up to fields above the critical value $h_{\mathrm{c}}=2$.

In a recent paper [4] we presented an approximate analytic expression for the dynamic spin correlation functions in $(q, \omega)$-space at $T=0$ and $h=0$, taking into account excitations from the (singlet) ground state to the spin-wave continuum of triplet states:

$$
G_{x x}(q, \omega)=2\left[\omega^{2}-E_{\mathrm{L}}^{2}(q)\right]^{-1 / 2} \Theta\left(\omega-E_{\mathrm{L}}(q)\right) \Theta\left(E_{\mathrm{U}}(q)-\omega\right) .
$$

Here $E_{\mathrm{L}}(q)=(\pi / 2)|\sin q|$ and $E_{\mathrm{U}}(q)=\pi|\sin (q / 2)|$. Our autocorrelation function $\phi_{x x}(\omega)$ is immediately found by integration over $q$. It shows a logarithmic divergence at $\omega=\pi / 2$, and the zero frequency limit is

$$
\phi_{x x}(\omega)=2 / \pi+\mathrm{O}(\omega)
$$

Obviously, for $h=0$,

$$
\phi_{z z}(\omega)=\phi_{x x}(\omega)
$$


For fields $h \geq h_{\mathrm{c}}$ Bethe's formalism yields the exact result (for $T=0$ ):

$$
\phi_{x x}(\omega)=\frac{1}{2}\left[1-\left(1+h-h_{\mathrm{c}}-\omega\right)^{2}\right]^{-1 / 2} \Theta\left(\omega-\left(h-h_{\mathrm{c}}\right)\right) \Theta\left(2+h-h_{\mathrm{c}}-\omega\right) .
$$

At the critical field $\phi_{x x}(0)$ diverges, whereas it vanishes for $h>h_{\mathrm{c}}$. For $0<h<h_{\mathrm{c}}$ finite-chain calculations suggest that the dominant contribution to $\phi_{x x}(0)$ again comes from excitations near $q=\pi$, as for $h=0$ and $h=h_{\mathrm{c}}$. Bethe's formalism allows for an approximate calculation of the lower boundary of that spin-wave continuum which contributes to $\phi_{x x}[5]$ :

$$
E_{L}(q)=2 D|\cos (q / 2) \sin (q / 2-\pi \sigma)|,
$$

where $D=(1-h / 2)(\pi / 2-1)+1$ and $\sigma$ is the magnetization, given by $\sigma=\pi^{-1} \arcsin (h / 2 D)$. Assuming that the spectral weight of $G_{x x}(q, \omega)$ above $E_{L}(q)$ still has a square root behaviour as in eq. (3) the $q$-integration yields

$$
\phi_{x x}(0)=2\left(4 D^{2}-h^{2}\right)^{-1 / 2} .
$$

At zero field $\phi_{x x}(0)=2 / \pi$ and at the critical field $\phi_{x x}(0)$ diverges. Essentially the same behaviour of $\phi_{x x}(0)$ as in eq. (7), has been found by Groen et al. [6] using a completely different approach.

Since an analytical treatment for finite temperatures seems to be out of reach for the time being, we also performed numerical calculations for finite chains. In fig. 1 the field dependence of $\phi_{x x}(0)=2 / \pi$ for a cyclic chain of 8 spins at $T=0.17$ (corresponding to $0.5 \mathrm{~K}$ for $\mathrm{CuSO}_{4}$ ) is compared with very recent experimental values for $T_{1}^{-1}$ obtained by Groen [7] and with eq. (7). [The geometry of these experiments was chosen such that the constant $A_{z}$ in our eq. (2) was zero.] Our results for higher $T$ are also in good agreement with the experimental data of ref. [3]. Details will be published elsewhere.

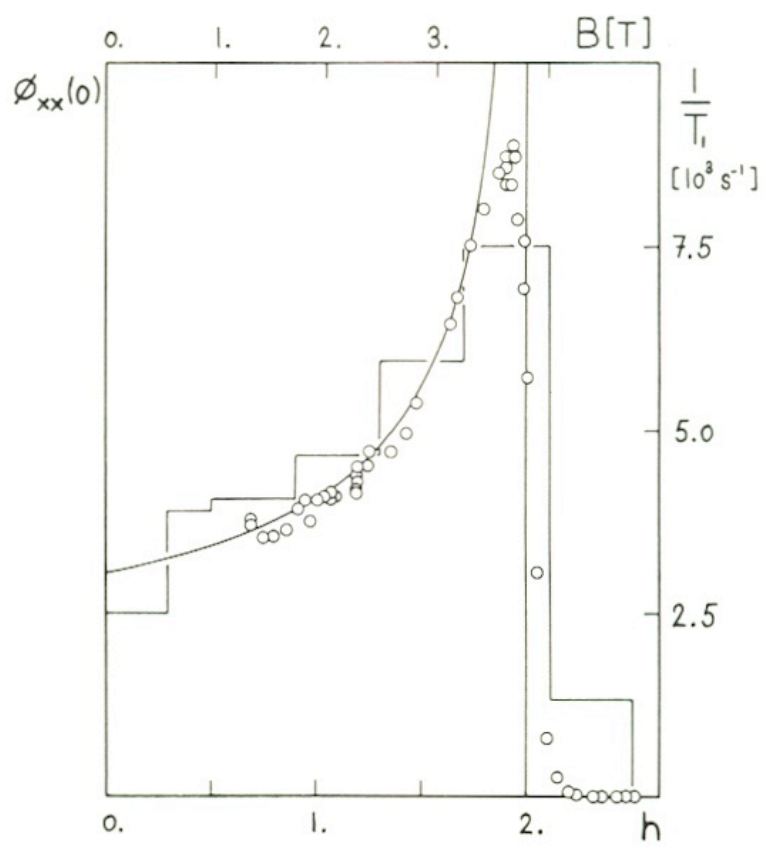

Figure 1. This figure shows the field dependence of the transverse autocorrelation function $\phi_{x x}(\omega=0)$. The histogram represents the result for a cyclic chain containing 8 spins at a reduced temperature $T=0.17$ and the continuous curve the result (7) of our spin-wave continuum approach at $T=0$. The circles denote experimental values of the inverse relaxation time $T_{1}^{-1}$ obtained by Groen (7) on $\mathrm{CuSO}_{4}$ for a geometry with $T_{1}^{-1} \propto \phi_{x x}(0)$. The magnetic field $B$ is given in tesla. Both theoretical curves are scaled independently in order to compare them directly with the data points. 


\section{Acknowledgments}

We are indebted to J. P. Groen for sending us his data prior to publication and allowing their inclusion in our figure. One of us (G. M.) acknowledges interesting discussions with H. W. Capel, J. H. H. Perk and N. J. Poulis and H. Thomas. This work was supported by the Swiss National Foundation. We have used a modified cmpj.sty style file.

\section{References}

1. L. S. J. M. Henkens, T. O. Klaassen and N. J. Poulis, Physica 94B (1978) 27.

2. P. M. Richards and F. Borsa, Sol. State Commun. 15 (1974) 135.

3. J. P. Groen, T. O. Klaassen and N. J. Poulis, Phys. Lett. 68A (1978) 381.

4. G. Müller, H. Beck and J. C. Bonner, Phys. Rev. Lett. 43 (1979) 75.

5. G. Müller, H, Beck and J. C. Bonner, same issue.

6. J. P. Groen, H. W. Capel, J. H. H. Perk, T. O. Klaassen and N. J. Poulis, Physica 97B (1979) 126.

7. J. P. Groen, private communication 